\title{
Stabilization of Energetic-Ion-Driven MHD Mode by ECCD in Heliotron J
}

\author{
K. Nagasaki ${ }^{1}$, S. Yamamoto ${ }^{1}$, S. Kobayashi ${ }^{1}$, K. Sakamoto ${ }^{1}$, Y. I. Nakamura ${ }^{2}$, Y. Nagae ${ }^{2}$, Y. \\ Sugimito $^{2}$, G. Weir ${ }^{3}$, N. Marushchenko ${ }^{4}$, T. Mizuuchi ${ }^{1}$, H. Okada ${ }^{1}$, T. Minami ${ }^{1}$, K. Masuda ${ }^{1}$, \\ S. Ohshima ${ }^{1}$, S. Konoshima ${ }^{1}$, Y. Nakamura ${ }^{2}$, H. Y. Lee ${ }^{2}$, L. Zang ${ }^{2}$, S. Arai, H. Fukushima ${ }^{2}$, H.

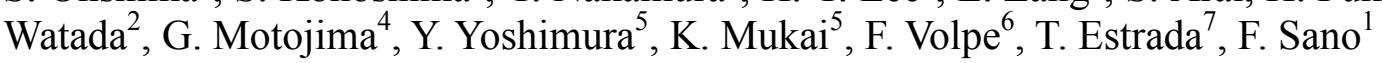 \\ ${ }^{1}$ Institute of Advanced Energy, Kyoto University, Japan \\ ${ }^{2}$ Graduate School of Energy Science, Kyoto Univ., Japan \\ ${ }^{3}$ University of Wisconsin, Madison, U. S. A. \\ ${ }^{4}$ Max-Planck-Institut für Plasmaphysik, EURATOM Association, Teilinstitut Greifswald, \\ Germany \\ ${ }^{5}$ National Institute for Fusion Science, Japan \\ ${ }^{6}$ Columbia University, New York, U. S. A. \\ ${ }^{7}$ Laboratorio Nacional de Fusión, EURATOM-CIEMAT, Spain
}

E-mail contact of main author: nagasaki@iae.kyoto-u.ac.jp

\begin{abstract}
Second harmonic electron cyclotron current drive (ECCD) has been applied in Heliotron J to stabilize magnetohydrodynamic (MHD) modes in Heliotron J. Localized EC current driven at central region modifies the rotational transform profile, $t / 2 \pi$, making a high magnetic shear. An energetic-ion-driven MHD mode of $80 \mathrm{kHz}$ has been fully stabilized by co-ECCD, and another mode of $90 \mathrm{kHz}$ has been stabilized by counter-ECCD when the EC current of a few kA is driven. Both co- and counter-ECCD is effective for the energetic-ion-driven MHD modes. An experiment of scanning the EC driven current shows that there is a threshold in magnetic shear to stabilize the energetic-ion-driven MHD mode.
\end{abstract}

\section{Introduction}

Understanding and control of magnetohydrodynamic (MHD) modes is an important issue for sustaining high-perfomance plasmas in toroidal fusion devices such as tokamaks and stellerator/heliotron $(\mathrm{S} / \mathrm{H})$ devices. Energetic alpha particles produced through deuterium-tritium fusion process and beam ions used for plasma heating, which have a velocity comparable with the Alfvén velocity, can interact resonantly with shear Alfvén waves during slowing-down process, and excite MHD instabilities, that is, Alfvén eigenmodes (AEs), resulting in enhanced radial transport of the energetic ions. Even a small fraction of alpha power loss in a burning plasma can seriously damage plasma facing components. Alfvén eigenmodes such as toroidal Alfvén eigenmode (TAE), global Alfvén eigenmode (GAE), energetic particle mode (EPM) and reversed shear Alfvén eigenmode (RSAE) has been experimentally obesrved in several $\mathrm{S} / \mathrm{H}$ devices [1]. Enhancement of energetic-ion transport by these instabilities has been reported as the deleterious effects [2]. On the excitation of AEs and their effects, the important factors are the confinement flux surface parameters such as radial profiles of the rotational transform $1 / 2 \pi$, magnetic shear and magnetic well/hill.

While much attention is focused on gaining a predictive capability for these instabilities in future devices through modeling and extrapolation of current experimental results, less attention has been paid to suppression and control techniques. A technical scheme of suppressing a particular type of Alfvénic modes is advantageous and required to develop. Such a control technique could be used to prevent excessive energetic-ion loss and vessel damage resulting from AEs. This technique also provides the opportunity to investigate the properties of specific instabilities. For example, formation of reversed shear configuration was studied in LHD plasmas with high $\beta$ or counter NBCD [3]. However controllability of 
rotational transform profile by external source and stabilization of MHD modes have been studied little.

Electron cyclotron heating $(\mathrm{ECH})$ and electron cyclotron current drive (ECCD) is recognized as a useful scheme for stabilizing MHD instabilities such as neoclassical tearing mode and Alfvén eigenmodes in tokamaks. ECH/ECCD is an ideal tool since it can provide highly localized EC current with a known location and good controllability. In the DIII-D tokamak, for example, a reversed shear AE was stabilized by applying an $\mathrm{ECH}$ near minimum of the magnetic safety factor in NB heated discharge with reversed-magnetic shear. [4][5] Localized ECH near the mode location stabilized reversed shear AE activity and results in improved fast ion confinement relative to discharges with ECH deposition on axis. ECCD is expected also in $\mathrm{S} / \mathrm{H}$ systems as an effective current drive scheme to suppress the non-inductive current and to tailor the rotational transform profile, particularly in low-shear devices.

The Heliotron $\mathbf{J}$ is an weak magnetic shear helical device, whose poloidal magnetic field is relatively lower than the toroidal magnetic field due to low rotational transform. External current driven at core region of a few $\mathrm{kA}$ can easily change the rotational transform profile compared to tokamaks and high-shear helical systems such as LHD. MHD modes such as interchange mode and GAE have been observed in NBI plasmas [6], and it has been also demonstrated that MHD activity of $m / n=2 / 1$ interchange mode is excited when the rotational transform increases from 0.48 to 0.50 by bootstrap current and NB driven current [7]. This means that the Heliotron $\mathrm{J}$ device is advantageous to study the effect of external non-inductive current on MHD stabilization. Research on external controllability of rotational transform gives us characteristics of MHD modes whose excitation depends on local rotational transform and the way how to stabilize them.

In this paper, we report experimental results on stabilization of energetic-ion driven MHD modes by ECCD in Heliotron J. The organization of the paper is as follows. Experimental setup and properties of ECCD is described in Sec. 2. Control of rotational tranform by ECCD is discussed in Sec. 3. Experimental results on stabilization of AEs by ECCD are shown in Sec. 4. Summary is given in Sec. 5.

\section{Experimental Setup}

\subsection{Heliotron J Device and 70GHz ECH/ECCD System}

Heliotron $\mathrm{J}$ is a medium-sized plasma experimental $\mathrm{S} / \mathrm{H}$ device [8][9]. The device parameters are the plasma major radius $R=1.2 \mathrm{~m}$, the averaged minor radius $a=0.1-0.2 \mathrm{~m}$, the rotational transform $\mathrm{l} / 2 \pi=0.3-0.8$, and the maximum magnetic field strength on the magnetic axis, $B=1.5 \mathrm{~T}$. The coil system is composed of an $L=1, M=4$ helical coil, two types of toroidal coils A and B, and three pairs of vertical coils. The magnetic configuration is possible to scan widely on Heliotron $\mathrm{J}$ by varying the current ratios in each coil, making it possible to investigate the properties of noninductive current including bootstrap current and EC driven current in a wide range of magnetic configurations.

Plasmas are produced and heated by a $70-\mathrm{GHz}$ second harmonic X-mode ECH and neutral beam injection. The focused EC beam radius of $1 / e^{2}$ power is $3 \mathrm{~cm}$ at the magnetic axis, smaller than the minor radius, $a \sim 17 \mathrm{~cm}$. The steerable mirror enables us to change the beam angle flexibly in the toroidal and poloidal directions, and the available $N_{\|}$ranges from -0.05 to 0.6 , limited by the chamber port size. Here $N_{\|}$is determined by the angle between axial magnetic field and EC wave vector under the vacuum condition. The new EC launcher is positioned between the straight and corner sections, and the X-mode fraction is more than $80 \%$, especially more than $90 \%$ for $0.1<N_{\|}<0.6$. Two tangential hydrogen beamlines of neutral beam injection (NBI, BL1:counter- and BL2:co-going directions) is used for NBI, 
which have a maximum acceleration voltage of $30 \mathrm{kV}$ and a total maximum power of $0.7 \mathrm{MW}$.

\subsection{Estimation of EC Driven Current}

ECCD experiments have been conducted using the upgraded launching system in Heliotron J. The total toroidal current is measured by Rogowski coils wound on the inner wall of the poloidal cross-sections at two different toroidal angles, that is, the corner and the straight sections. An ECH power of $260 \mathrm{~kW}$ is injected up to $140 \mathrm{msec}$. An $L / R$ time is about 100 $200 \mathrm{msec}$ for the Heliotron J plasma parameters, where $L$ and $R$ are the plasma inductance and resistance. In the experiment reported here, the measured toroidal current became saturated within the pulse length for $I_{\mathrm{p}}<3 \mathrm{kA}$. The EC current is experimentally evaluated in ECH plasmas without using theoretical input for bootstrap current. In ECH plasmas, the toroidal current is composed of the bootstrap current and the EC current. These currents can be separated by comparing the experimental results obtained for clockwise and counter-clockwise magnetic fields, since the flow direction of the bootstrap current, which is proportional to $\mathbf{B} \times \nabla B$ drift, is reversed by reversing the magnetic field, while that of the EC current associated with the $B$ strength is not. The ECCD experiment in Heliotron $\mathrm{J}$ showed that the EC driven current strongly depended on the magnetic field configuration [11][12][13], suggesting that ECCD is determined by the balance between the Fisch-Boozer effect [14] and the Ohkawa effect [15]. We also demonstrated that net zero current state was maintained by cancelling the bootstrap current with ECCD [11]. Experimental research on ECCD has been performed in several helical systems, showing that the ECCD efficiency was found to be similar [16].

The EC driven current experimentally measured is compared with a theoretical simulation using the TRAVIS ray tracing code. TRAVIS is a ray tracing code for ECH/ECCD and ECE diagnostics in arbitrary 3D magnetic configurations [17]. The code was successfully tested and applied for W7-X [18] and ITER [19]. For ECH/ECCD calculations, the absorption is decomposed into the contributions from trapped and passing electrons by analyzing during integration along the resonance line. The energy range of electrons responsible for absorption is also calculated. The resonant harmonics, which may participate in cyclotron interaction, are defined in the code automatically by analyzing the magnetic configuration. The CD efficiency
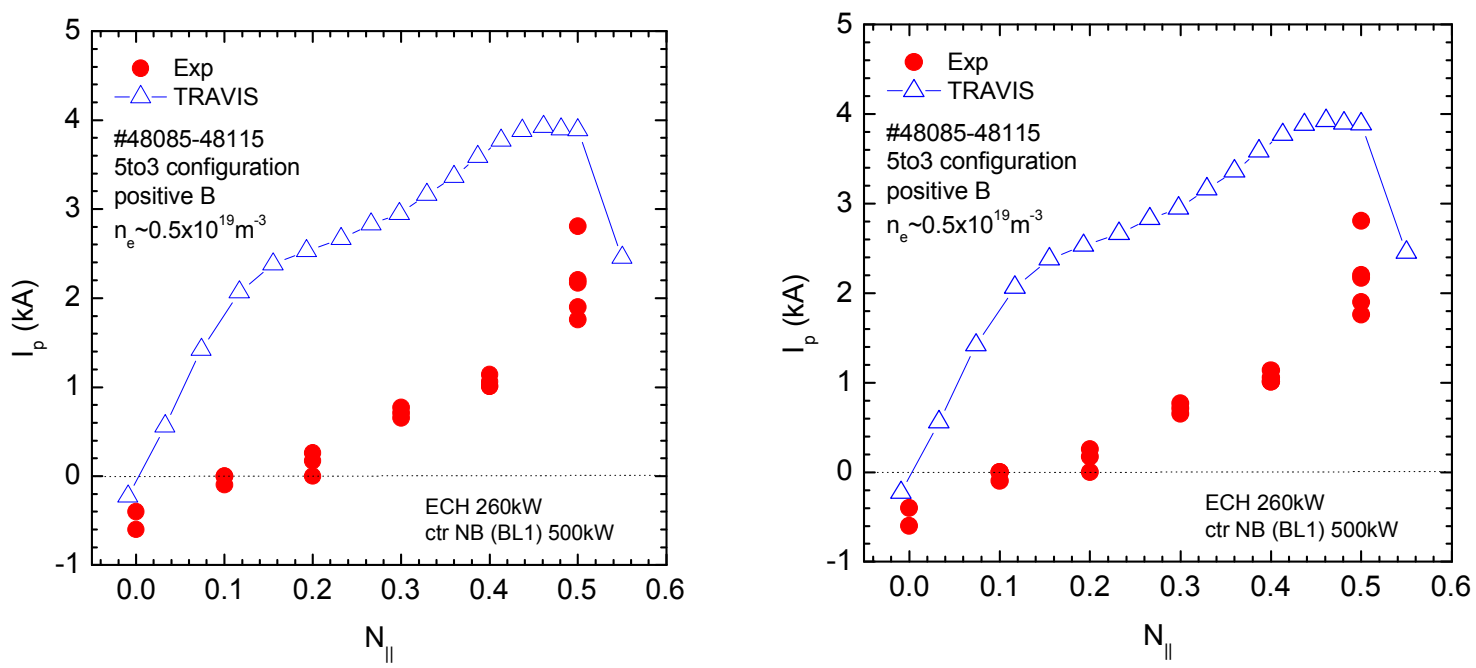

FIG. 1. Measured toroidal current in ECH+NBI plasmas as a function of parallel refractive index, $N_{\|}$, under (a) normal magnetic field condition and (b) reversed magnetic field condition. The counter NBI only is applied for the reversed magnetic field, and the co- and counter NBIS are applied for the normal magnetic field 
is calculated by applying the adjoint approach with parallel momentum conservation (pmc) taking into account. Quantitative agreement is focused between experimental and theoretical results in ECH plasmas for several different configurations. The trapped electrons have a significantly important role to determine the current drive efficiency. As the EC power is deposited at the ripple bottom, the power fraction absorbed by trapped particles increases, leading to the reduction in $\mathrm{EC}$ driven current.

Figure 1 shows the $N_{\|}$dependence of the EC driven current in ECH+NBI plasmas. The theoretical EC driven current calculated by TRAVIS code is also plotted. We define that the current flowing in the Fisch-Boozer direction is positive. The measured toroidal current is composed of EC driven current, NB driven current and bootstrap current. In these configurations, the bootstrap current is low, $I_{\mathrm{BS}} \sim 0 \mathrm{kA}$, consistent with a neoclassical theory including radial electric field effect. From the comparison between the measured toroidal current and the TRAVIS code result, the NB current is about $2 \mathrm{kA}$ and $1 \mathrm{kA}$ for the normal and reversed magnetic field conditions, respectively.

In ECH plasmas, the radiation temperature measured with a heterodyne radiometer has a peaked profile reflecting the bulk electron temperature for small $N_{\|}, N_{\|}=0.0-0.2$. As $\mathrm{N} \|$ increases, on the other hand, the radiation temperature drastically increases, having a flat profile. Since the optical thickness is gray, $\tau \sim 1$, non-thermal electrons are more generated or confined for larger $N_{\|}$, contributing to driving the EC current. This can be explained by the energy range of resonant electrons. According to the TRAVIS code, the energy of resonant electrons interacting with the EC waves shifts it center from 1.4 to 2.0 as $N_{\|}$increases from 0.0 to 0.6 , and it has a narrow energy range around $u / u_{\mathrm{th}} \sim 1.4$ for $N_{\|}=0.0$, while it is wider with an increase of $N_{\|}$, that is, the energy range is $0.9<u / u_{\text {th }}<2.7$.

\section{Control of Rotational Transform Profile by ECCD}

$\mathrm{EC}$ driven current of a few $\mathrm{kA}$ is enough to modify the rotational transform profile in Heliotron $\mathrm{J}$ if it flows at the central region. Although the total amount of EC driven current is rather smaller than that in tokamaks, it is comparable to other non-inductive current such as bootstrap current and NB current. Figure 2 illustrates the rotational transform profiles with ECCD. The rotational transform in vacuum is 0.525 , and the EC driven current profile is calculated by the TRAVIS code. The total current at $N_{\|}=0.4$ is $2.9 \mathrm{kA}$ at the line-averaged electron density of $n_{\mathrm{e}}=3.0 \times$ $10^{19} \mathrm{~m}^{-3}$, and the electron temperature of $T_{\mathrm{e}}(0)=0.8 \mathrm{keV}$. The magnetic field strength is $1.25 \mathrm{~T}$ so that the co EC current can be driven around magnetic axis. It is seen that the co-ECCD reduces the central rotational transform, making a high negative magnetic shear, vice versa.

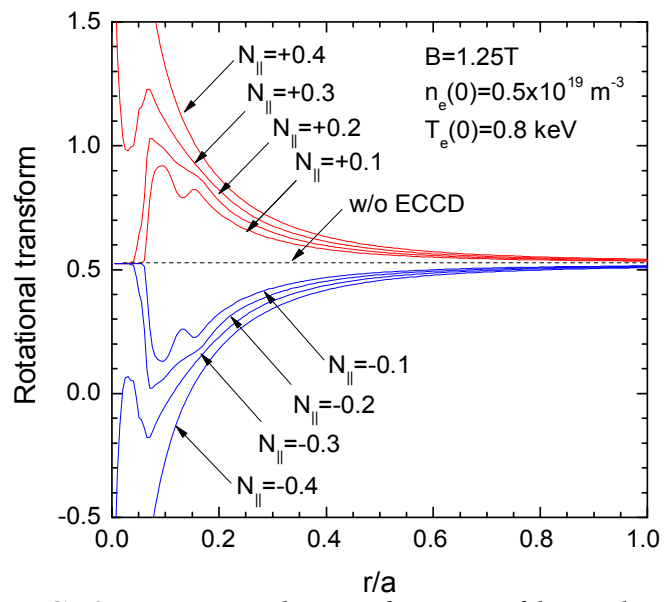

FIG. 2. Rotational transform profile with ECCD in Heliotron $J$. The positive and negative $N_{\|}$ correspond to co- and counter-ECCD, respectively.

The time evolution of rotational transform is calculated by the following one-dimensional diffusion equation for toroidal current [20], 


$$
\mu_{0} \frac{d I_{p}}{d t}=\frac{1}{2 \pi r} \frac{\partial}{\partial r}\left\{\frac{1}{\sigma} \frac{\partial}{\partial r}\left(I_{p}-I_{N I}\right)\right\}
$$

where $I_{\mathrm{p}}=I_{\mathrm{p}}(\mathrm{r}, \mathrm{t})$ and $I_{\mathrm{NI}}=I_{\mathrm{NI}}(\mathrm{r}, \mathrm{t})$ are the toroidal current and the non-inductive current in a magnetic surface, respectively, $\mu_{0}$ is the magnetic permeability in vacuum, $\sigma$ is the electrical conductivity. The boundary conditions are given as $I_{\mathrm{p}}(0, \mathrm{t})=0, I_{\mathrm{p}}(\mathrm{a}, \mathrm{t})=I_{\mathrm{p} 0}\left\{1-\exp \left(-\mathrm{t} / \tau_{\mathrm{d}}\right)\right\}$ where $I_{\mathrm{p} 0}$ and $\tau_{\mathrm{d}}$ are determined by simulating experimental results. Figure 3 show an example of time evolution of rotational transform profile in standard configuration of $\mathrm{l} / 2 \pi=0.56$. The EC current is assumed to have a Gaussian profile with the peaked position of $r / a=0.25$ and the $1 / \mathrm{e}$ radius of 0.2 . The characteristic diffusion time, $\tau_{\mathrm{d}}$, is given by $100 \mathrm{msec}$, and it takes a current diffusion time to reach a steady state after applying ECCD. The toroidal electric field is excited at core region, reducing the central rotational transform at initial phase, but it gradually approaches to the steady state. This calculation suggests that the rotational transform changes in time, and approaches nearly the steady state within the experimental discharge time $(\sim 100 \mathrm{msec})$.

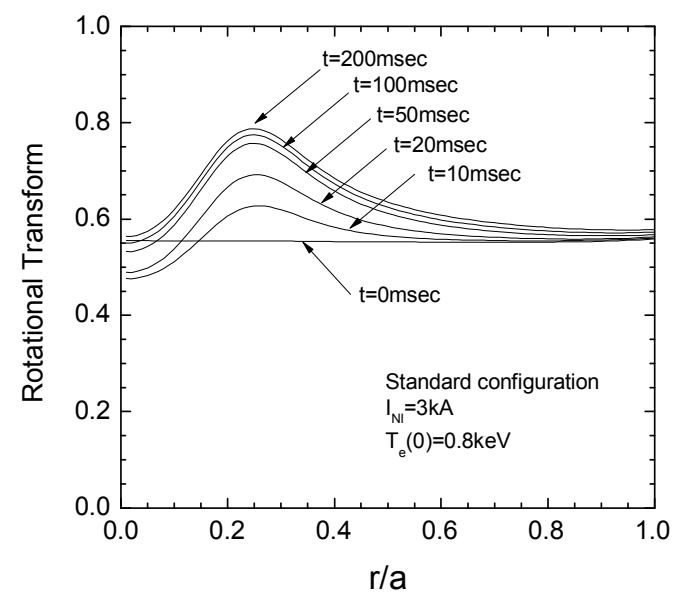

FIG. 3. Time evolution of rotational transform profile calculated by a current diffusion equation.

\section{Stabilization of Energetic-Ion-Driven MHD Modes by ECCD}

Coherent modes in high frequency range of $f>30 \mathrm{kHz}$ excited by energetic ions are often observed with magnetic probes, a beam emission spectroscopy (BES), a soft-X ray array and a reflectometer in NBI-heated Heliotron J plasmas. The feature of excited AEs in helical systems depends on the flux surface parameters such as rotational transform, its shear, and magnetic well/hill (See Ref. [21]). Typically observed are GAE and EPM. The magnetic fluctuation amplitudes of these modes are on the order of $b_{\theta} / B_{\mathrm{t}}=10^{-6}-10^{-5}$ at the magnetic probe position. In shearless $\mathrm{S} / \mathrm{H}$ systems, GAEs are generally excited just below the minimum frequency of shear Alfvén continuum since the GAEs can avoid the shear Alfvén spectrum and do not suffer from continuum damping.
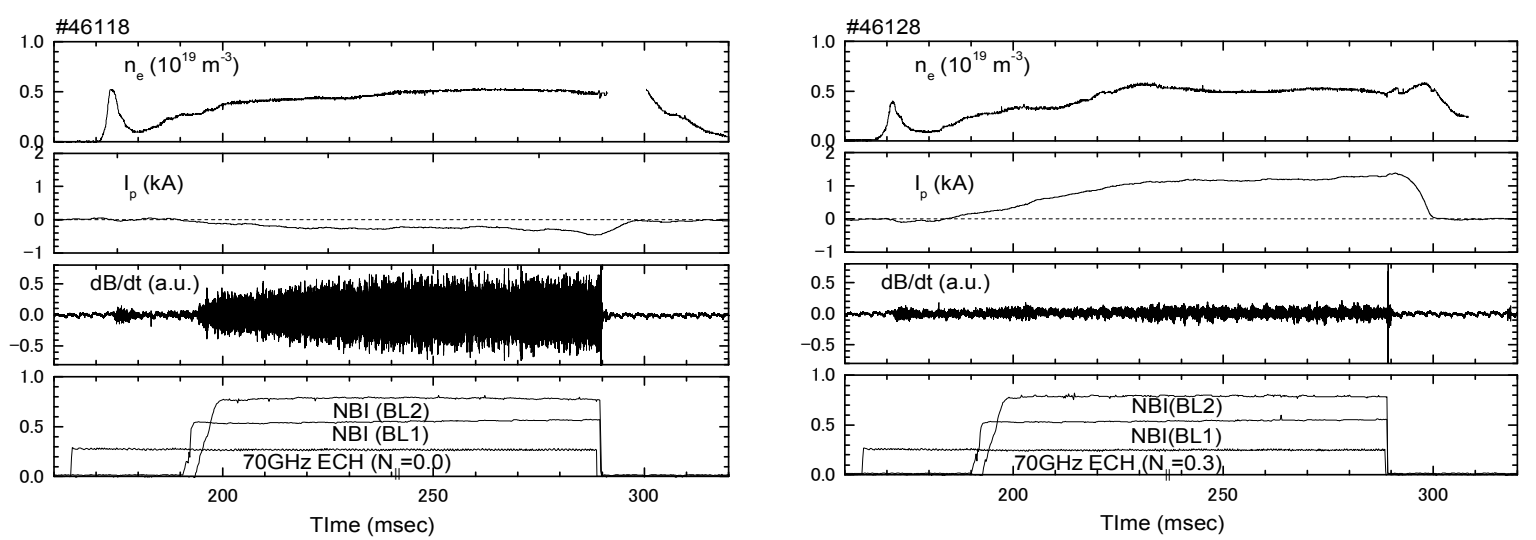

$F I G$. 4. Time evolution of ECH+NBI plasmas (a) without ECCD and (b) with ECCD. The ECH power is $0.3 \mathrm{MW}$, and the co- and counter-NBI powers are $0.8 \mathrm{MW}$ and $0.6 \mathrm{MW}$, respectively. 
Effect on MHD modes has been experimentally studied by the $70-\mathrm{GHz}$ second harmonic $\mathrm{X}$-mode ECCD. Figure 4 shows the time evolution of ECH+NBI plasmas. The magnetic configuration and magnetic field strength in vacuum are set as in Fig. 1(b), and the balanced NB power is injected. In this discharge, the EC current is driven in the direction to decrease the rotational transform. The operating electron density is $0.5 \times 10^{19} \mathrm{~m}^{-3}$, which is much lower than the second harmonic X-mode cut-off, $3.0 \times 10^{19} \mathrm{~m}^{-3}$, so that the EC current is localized due to less refraction effect. The electron density is so low that $\beta$ effect $(\beta \sim 0.1 \%)$ on rotational transform is negligible compared to ECCD. The TRAVIS code results indicate that the EC power is deposited at $r / a<0.25$, and does not change much for $0.0<N_{\|}<0.4$, meaning that NB current and bootstrap current is almost constant. For $N_{\|}=0.0$, non-inductive current of $-0.5 \mathrm{kA}$ flows, consisting of bootstrap current and NB current.

(a)

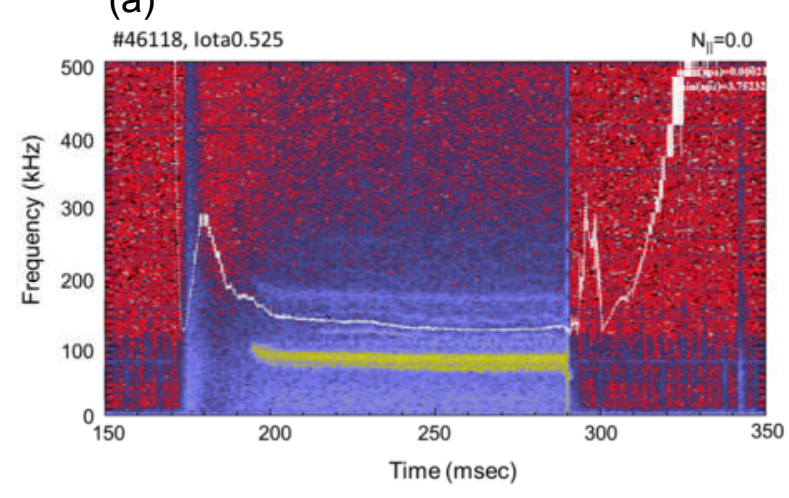

(b)

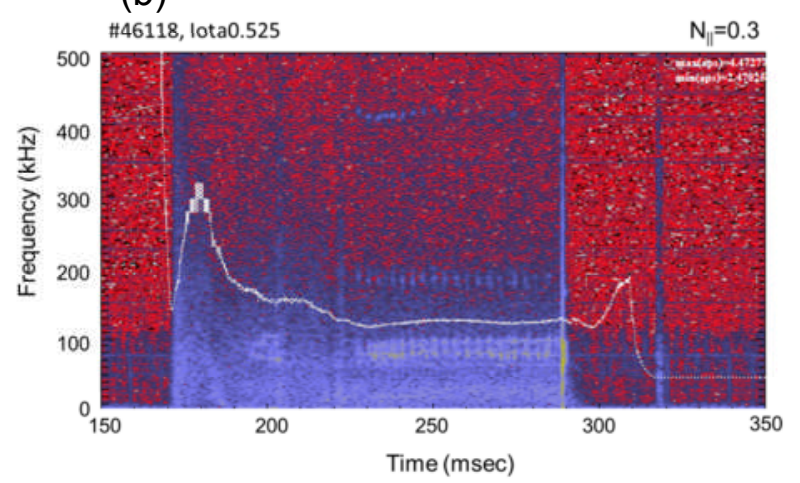

FIG. 5 Fourier spectrograms of magnetic fluctuation for (a) $N_{\|}=0.0$ and (b) $N_{\|}=0.3$. The discharges are the same as in Fig. 4.

As shown in Fig. 5, FFT analysis of Mirnov coil signals shows that a coherent mode of $\mathrm{m} / \mathrm{n}=$ $4 / 2$ is excited at $f=80 \mathrm{kHz}$ which rotates in the ion diamagnetic direction. This mode is fully suppressed when applying the ECCD. Density fluctuation measurement using a multi-channel beam emission spectroscopy [22] reveals that the mode is localized around $r / a \sim 0.6$ as shown in Fig. 6 This mode has been successfully suppressed for $N_{\|}=-0.3$, where the EC current of $2.4 \mathrm{kA}$ flows in the direction to decrease the rotational transform. Since the mode is localized in minor radius $\mathrm{r} / \mathrm{a} \sim 0.6, \mathrm{EPM}$ is a possible candidate for the observed mode. A resonant Alfvén eigenmode, EPM, is excited under finite high-energy particle pressure, and its frequency is determined by characteristic velocity of high energy particles. This mode suffers from continuum spectrum damping, but it is destabilized when the driving term due to high energy ions exceeds the continuum spectrum damping. The EPM is excited in a localized position where the above condition is satisfied. It usually exhibits bursting characteristics having rapid frequency chirping, intermittent amplitude modulation and spectral separation due to nonlinear effect. Since there was no such frequency chirping in the observed mode, further investigation is necessary to clarify whether it is the EPM or not by measuring the density and rotational transform profiles.

In typical shots reported here, $\mathrm{ECH}$ is injected from the initial phase of the discharge to obtain quasi-steady state for toroidal current. In such discharges, the toroidal current gradually increases with a current diffusion time scale. However, in some shots, the increase in EC current is delayed, and the relationship between the mode amplitude and the toroidal current can be studied. Figure 7 show a dynamic response of the AE mode amplitude to the toroidal current. In this shot, the toroidal current increases after the AE is excited by NB. The mode amplitude increases up while the EC current is kept zero, and then it is gradually reduced and 


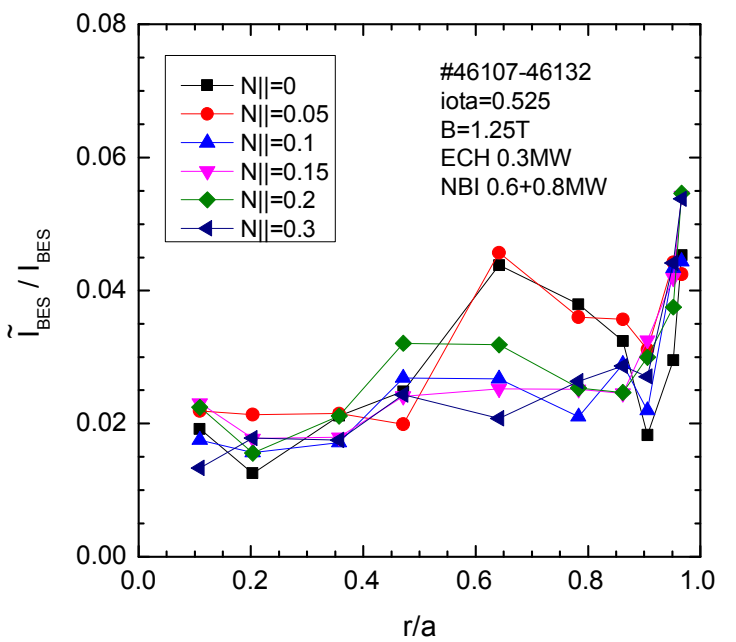

FIG. 6 Density fluctuation profiles in $E C H+N B I$ plasmas measured with beam emission spectroscopy. A coherent mode excited at r/a 0.6 is suppressed by ECCD of $N_{\|}>0.1$

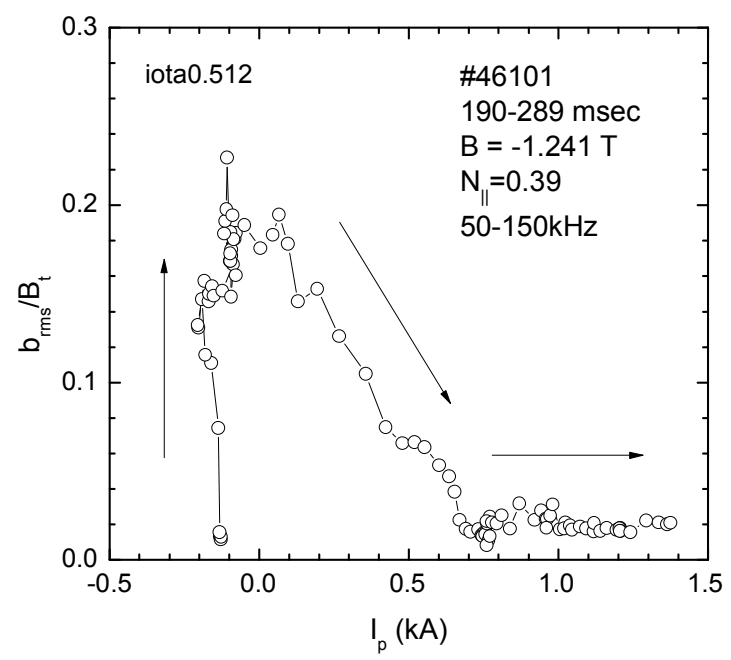

FIG. 7. Magnetic fluctuation amplitude of coherent mode as a function of plasma

reaches a mode level of ECH plasmas as the EC current increases. Since the reduction in mode amplitude smoothly happen, the mode suppression does not have transition property.

Co-ECCD has been also performed in $\mathrm{ECH}+\mathrm{NBI}$ plasmas in the magnetic configuration of $\imath / 2 \pi=0.56$. Only the counter beam line of $500 \mathrm{~kW}$ is injected for NBI. An AE of $90 \mathrm{kHz}$ is observed in magnetic probe signals for $\mathrm{N} \|=0.0$. According to the BES measurement, the coherent mode of $100 \mathrm{kHz}$ which has a high coherence with the magnetic probe signal is excited in a rather wide region, suggesting that the mode is a GAE. When the EC current of about $1 \mathrm{kA}$ is applied in the direction of increasing the central rotational transform, the mode amplitude gradually decreases, and reaches the same level as that in the ECH plasmas.

The stabilizing mechanism may be related to formation of magnetic shear. The effect of magnetic shear on MHD modes is investigated by scanning the EC driven current. Figure 8 shows the magnetic shear dependence of the mode amplitude. The magnetic configuration is the same as in Fig. 1(b). The magnetic shear is estimated at $r / a=$ 0.6 where the mode is excited. The calculated EC driven current monotonically increases from $-0.3 \mathrm{kA}$ to $2.4 \mathrm{kA}$ in the range of $-0.3<$ $N_{\|}<0.0$. For the EC driven current more than $2.0 \mathrm{kA}$, the mode amplitude is completely suppressed to the level of ECH-only phase. This result indicates that there is a threshold in magnetic shear to stabilize the AE.

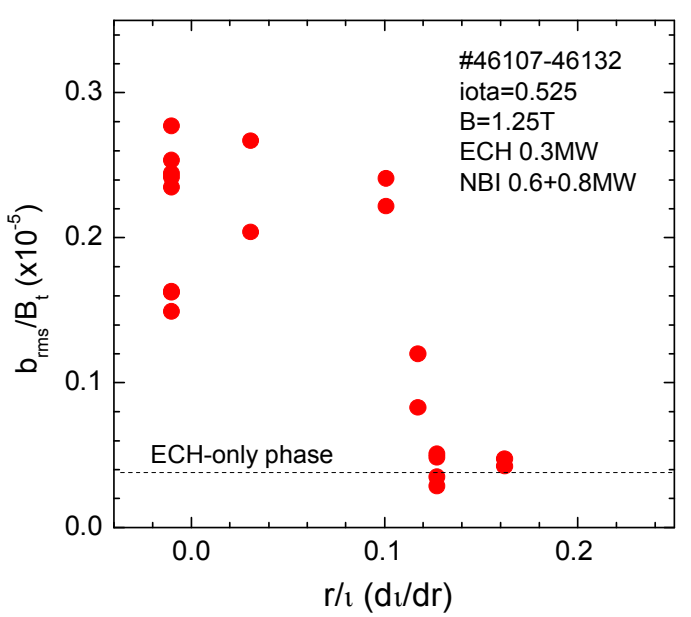

Fig. 8 Magnetic shear dependence of $A E$ mode amplitude in couter-ECCD stabilization. The experimental condition is the same as in Fig. 2.

\section{Summary}

ECCD experiments have been made for stabilization of energetic-ion-driven MHD modes in Heliotron J. The theoretical analysis show that the EC current of a few kA driven at the 
central region modifies the rotational transform profile from a shearless flat one in vacuum state into a high-shear one in Heliotron J. The ECCD has been applied to ECH+NBI plasmas in which AEs are excited by energetic ions. The energetic-ion-driven MHD modes have been fully stabilized by centrally localized second harmonic $70-\mathrm{GHz} \mathrm{X}$-mode ECCD. In the magnetic configuration of $\mathrm{v} / 2 \pi=0.512$ and 0.525 , the $\mathrm{AE}$ of $80 \mathrm{kHz}$ is stabilized by the counter-ECCD which forms a positive magnetic shear. Since the excited mode is localized at $\mathrm{r} / \mathrm{a} \sim 0.6$, the change in the local magnetic shear contributes to the mode stabilization. The $N_{\|}$ scan indicates that the EPM is stabilized when the magnetic shear exceeds a critical threshold. This tendency is consistent with the excitation theory of AEs. In the magnetic configuration of $\mathrm{v} / 2 \pi=0.56$, another AE of $90 \mathrm{kHz}$ has been stabilized by the co-ECCD which forms a negative magnetic shear at core region. Since measurement of the plasma profiles and rotational transform profile is not yet available, detailed comparison with the AE excitation theory is left for future. Further study on excitation and suppression of the AEs will be also performed experimentally in future by adjusting the amplitude and the location of ECCD in Heliotron J.

\section{Acknowledgments}

The authors are grateful to Heliotron $\mathbf{J}$ staff for conducting the experiments. This work is performed with the support and under the auspices of the Collaboration Program of the Laboratory for Complex Energy Processes, IAE, Kyoto University, and the NIFS Collaborative Research Program (NIFS08KOAR010, NIFS10KUHL030), the NIFS/NINS project of Formation of International Network for Scientific Collaborations, and the Grant-in-Aid for Scientific Research, MEXT.

\section{References}

[1] TOI, K., et al., Plasma Physics. Control. Fusion 53 (2011) 024008

[2] ISOBE, M., et al., Nucl. Fusion 50 (2010) 084007

[3] TOI, K., et al., Phys. Rev. Lett. 105 (2010) 145003

[4] VAN ZEELAND, M. A., Plasma Phys. Control. Fusion 50 (2008) 035009

[5] VAN ZEELAND, M. A., Nucl. Fusion 49 (2009) 065003

[6] YAMAMOTO, S., et al., Fusion Sci. Technol. (2007)

[7] MOTOJIMA, G., et al., Plasma and Fusion Res. 3 (2008) S1067.

[8] WAKATANI, M., et al., Nucl. Fusion 40 (2000) 569.

[9] OBIKI, T., et al., Nucl. Fusion 41 (2001) 833.

[10]NAGASAKI, K., et al., Contrib. Plasma Phys. 50, No. 6-7 (2010) 656.

[11]MOTOJIMA, G., et al., Nucl. Fusion 47 (2007) 1045.

[12]NAGASAKI, K., et al., Nucl. Fusion 50 (2010) 025003.

[13]NAGASAKI, K. et al., Nucl. Fusion 51 (2011) 103035

[14]FISCH, N. J. and BOOZER, A., Phys. Rev. Lett. 45 (1980) 720.

[15]OHKAWA, T., General Atomics Report GA-A13847 (1976).

[16]NAGASAKI, K., et al., Plasma Fusion Res. 3 (2008) S1008

[17] MARUCHCHENKO, N. B. et al., Plasma Fusion Res. 2 (2007) S1129

[18]ERCKMANN, V., et al., Fusion Sci. Technol. 52 (2007) 291

[19]MARUSHCHENKO, N.B., et al., 49 (2009) 129801

[20]WATANABE, K. Y., et al., J. Plasma Fusion Res. SERIES 5 (2002) 124-130.

[21]YAMAMOTO, S., et al., this conference, EX/5-2

[22]KOBAYASHI, S., et al., Rev. Sci. Instrum. 83 (2012) 10D535 